\title{
Combined parametric mapping allows discrimination of disease activity in myocarditis
}

\author{
Johannes Schüler ${ }^{1 *}$, André Rudolph ${ }^{1}$, Luisa M Schmacht ${ }^{1}$, Florian von Knobelsdorff-Brenkenhoff ${ }^{1}$, \\ Matthias A Dieringer ${ }^{1}$, Andreas Greiser ${ }^{2}$, Peter Kellman², Marcel Prothmann', Jeanette Schulz-Menger ${ }^{1}$ \\ From 19th Annual SCMR Scientific Sessions \\ Los Angeles, CA, USA. 27-30 January 2016
}

\section{Background}

Noninvasive detection of myocarditis is a unique feature of Cardiovascular Magnetic Resonance (CMR), but conventional CMR-imaging suffers from different challenges including imaging quality. It has already been shown, that T1 mapping has the potential to identify acute myocarditis $[1,2]$.

We aim to differentiate the course of disease and to identify the acute stage by combining mapping techniques.

\section{Methods}

Eighteen patients with clinically defined acute myocarditis were prospectively examined three times during follow up using a $1.5 \mathrm{~T}$ Scanner (FU0: within 2 weeks of symptoms onset, FU1: 5 weeks FU2: after 6 months). They were compared to a healthy sex- and age matched control group. Severity of disease was rated in NYHA and CCS score. Left ventricular (LV) volumes, mass and function were assessed using steady-state free precession (SSFP) cine imaging in short axis. Parametric mapping was applied using T2 maps in three short axis (TE $1.15 \mathrm{~ms}$, ST $6 \mathrm{~mm}$, SSFP) and native T1 mapping using modified look-locker inversion recovery (MOLLI 5(3)5) sequence (TE $1.11 \mathrm{ms,}$ ST $6 \mathrm{~mm}$ ) in a basal and a midventricular slice. Furthermore, T1 mapping was performed during contrast media wash-out (Gadobutrol $0.15 \mathrm{mmol} / \mathrm{kgBW}$ ) at minute $1,3,5,7,10$. Late gadolinium enhancement (LGE) was performed after minute 12 for myocardial fibrosis detection. Blood collection was done for haematocrit assessment. Data were analyzed using CVI42 (circle cardiovascular imaging Inc., Calgary, Canada), Mann-Whitney U test was used.

${ }^{1}$ Charité Medical Faculty and HELIOS clinics, Working group Cardiovascular MRI, Berlin, Germany

Full list of author information is available at the end of the article

\section{Results}

All 18 patients and healthy volunteers completed all examinations (age $30.06 \pm 10.9$ vs. $29.72 \pm 9.93$ years, 14 male and 4 female, respectively). NYHA score decreased from $1.8 \pm 0.95$ (FU0) to $0.5 \pm 0.5$ (FU2), CCS score decreased from 2.3 (FU0) to 0.3 (FU2).

14(78\%) patients showed focal fibrosis (LGE pos).

Global T2 relaxation times decreased during follow up ( $\mathrm{p}<0.001$ from FU0 to FU2). ROC analysis revealed a area under the curve of 0.9 for cutoff $51.92 \mathrm{~ms}$ (sensitivity $89 \%$, specifity $83 \%$ ) to differentiate diseased from healthy volunteers. Global native T1 remains stable (FU0 to FU2, $\mathrm{p}=0.311$ ), however, native T1 was significantly higher in patients than in healthy volunteers $(\mathrm{p}=0.003)$. Difference in $\mathrm{T} 1$ during contrast media washout between patients and healthy volunteers was significant at minute 3 at FU0 (321 \pm 37 ms vs. $361 \pm 31 \mathrm{~ms}, \mathrm{p}=0.001)$. Global ECV was increased at FU0 $(\mathrm{p}=0.021)$ and normalized at FU2 $(\mathrm{p}=0.164)$ compared to control group. See table 1 for details.

\section{Conclusions}

T1 mapping allows the identification of diseased in comparison to healthy volunteers. Additional use of T2 mapping enables to detect disease acuity and so far to identify reversibility. Further follow up examinations would be necessary to identify its prognostic values. Parametric mapping may replace parts of conventional imaging techniques.

\footnotetext{
Authors' details

${ }^{1}$ Charité Medical Faculty and HELIOS clinics, Working group Cardiovascular MRI, Berlin, Germany. ${ }^{2}$ Siemens Healthcare, Erlangen, Germany. ${ }^{3}$ Laboratory of Cardiac Energetics, National Institutes of Health/NHLBI, Bethesda, MD, USA.
} 
Table 1 Time course of LV ejection fraction and parametric mapping during follow up compared to healthy control group

\begin{tabular}{ccccc}
\hline & FU 0 acute phase & FU1 5 weeks & FU2 6 months & Healthy control group \\
\hline ejection fraction (\%) & $58.5 \pm 6.7(p=0.133)$ & $60.37 \pm 6.1(p=0.548)$ & $60.03 \pm 4.4(p=0.658)$ & $61.32 \pm 3.7$ \\
Global T2 time (ms) & $55.1 \pm 2,6(p<0.001)$ & $52.2 \pm 1.4(p=0.012)$ & $51.2 \pm 2(p=0.429)$ & $50.6 \pm 1.9$ \\
Native T1 time (ms) & $1015 \pm 36(p=0.003)$ & $1003 \pm 27(p=0.015)$ & $1001 \pm 31(p=0.043)$ & $978 \pm 32$ \\
Global ECV & $0.259 \pm 0.02(p=0.021)$ & $0.258 \pm 0.02(p=0.019)$ & $0.256 \pm 0.03(p=0.164)$ & $0.243 \pm 0.02$ \\
\hline
\end{tabular}



Published: 27 January 2016

\section{References}

1. Hinojar R, et al: $T 1$ mapping in myocarditis.... Expert Rev Cardiovasc Ther 2015.

2. Ferreira VM, et al: [Native T1-mapping ....] J Cardiovasc Magn Reson 2014. 